\title{
Lanthanide Coordination Polymer Nanosheet Aggregates: Solvothermal Synthesis and Downconversion Luminescence
}

\author{
Rui-Rui Su, ${ }^{1}$ Peng Tao, ${ }^{1}$ Yi Han, ${ }^{2}$ Cheng-Hui Zeng, ${ }^{1}$ and Sheng-Liang Zhong ${ }^{1}$ \\ ${ }^{1}$ Key Laboratory of Functional Small Organic Molecule, Ministry of Education and Jiangxi's Key Laboratory of Green Chemistry, \\ College of Chemistry and Chemical Engineering, Jiangxi Normal University, Nanchang 330022, China \\ ${ }^{2}$ School of Chemistry and Chemical Engineering, Sun Yat-Sen University, Guangzhou 510275, China
}

Correspondence should be addressed to Cheng-Hui Zeng; chenghuizeng@qq.com and Sheng-Liang Zhong; slzhong@jxnu.edu.cn

Received 23 February 2016; Accepted 10 April 2016

Academic Editor: Margarida Amaral

Copyright (C) 2016 Rui-Rui Su et al. This is an open access article distributed under the Creative Commons Attribution License, which permits unrestricted use, distribution, and reproduction in any medium, provided the original work is properly cited.

\begin{abstract}
A lanthanide coordination polymers (CPs) nanostructure (1) has been synthesized via a facile template-free solvothermal strategy using DMF as solvent and 2-methyl benzoic acid (2-MeBAH) as ligands. The products are characterized by powder X-ray diffraction (PXRD), scanning electron microscopy (SEM), elemental analyses (EA), and downward luminescence. Product 1 built from $\mathrm{Tb}^{3+}$ and 2-MeBAH has one-dimensional structure which is connected by trinuclear second building units (SBUs). Downward luminescence shows that sample 1 exhibits characteristic transitions of the $\mathrm{Tb}^{3+}$ ion at $489,544,583$, and $621 \mathrm{~nm}$, and the strongest peak is at $544 \mathrm{~nm}$ ascribed to the transition of ${ }^{5} \mathrm{D}_{4} \rightarrow{ }^{7} \mathrm{~F}_{5}$ of $\mathrm{Tb}^{3+}$
\end{abstract}

\section{Introduction}

Lanthanide coordination polymer $(\mathrm{CP})$ is an important class of organic-inorganic hybrid materials formed by the coordination of lanthanide ions with organic linkers. They have shown a number of promising applications; these applications are ascribed to the best properties of organic and inorganic components and decrease their drawbacks by a synergic effect [1]. In order to get functional lanthanide materials, appropriate synthetic strategies are critical $[2,3]$. Compared with first-row transition metal ions, lanthanide ions usually display high coordination number and variable coordination geometries, and they will make lanthanide ions become excellent spacers in assembling fascinating coordination polymers [4]. As a subset of such materials, lanthanide CPs continue to attract an escalating attention, owing to their unique optical, electronic, and magnetic properties [5-7]. In addition, nanosheet materials have been of continuous research interests in material science, because of their unique properties in the areas of catalysis, drug delivery, and biosensors. Herein, our group has successfully prepared terbiumbased CP nanosheet aggregates, with downward green-light emission while excited by violet light.
In modern chemistry and materials science, synthesis of uniform nanosheet materials with controllable size and well-defined morphologies remains a research focus due to their superior properties of large specific area, low effective density, and good permeation [8]. Thereby, hollow spherical materials constructed by the nanosheet possess widespread potential applications in various fields, including catalysts, adsorbents, photonic devices, sensors, drug-delivery carriers, disease diagnosis agents, waste removal, and chemical reactors [9]. In recent years, a variety of synthetic routes have been developed for the preparation of hollow spheres $[10,11]$ such as solvothermal method, hydrothermal method, precipitation method, spray pyrolysis method, and templateassisted method. Generally, the solvothermal method is used widely, because the solvent controlling effect is versatile.

As well known, lanthanide coordination polymers have been widely applied in luminescent devices $[12,13]$. However, compared with other morphologies of sphere, nanorod, and nanotube, the nanosheet of rare earth coordination polymers which are constructed into aggregates of hollow spheres morphologies has received much attention. Apart from possessing a collection of advantages, such as low weight and saving materials and costs, hollow sphere optical materials 


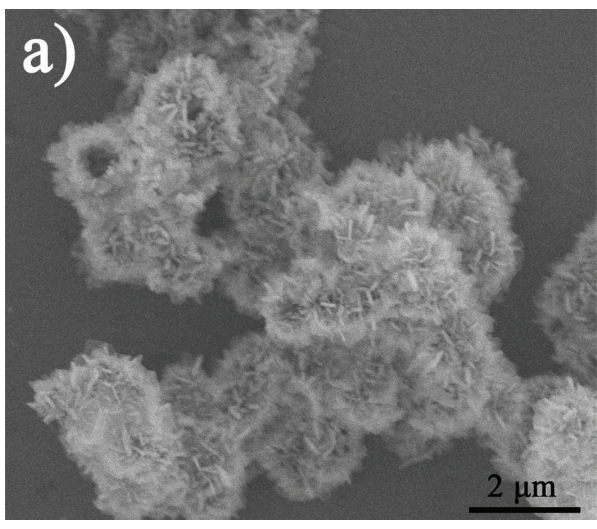

(a)

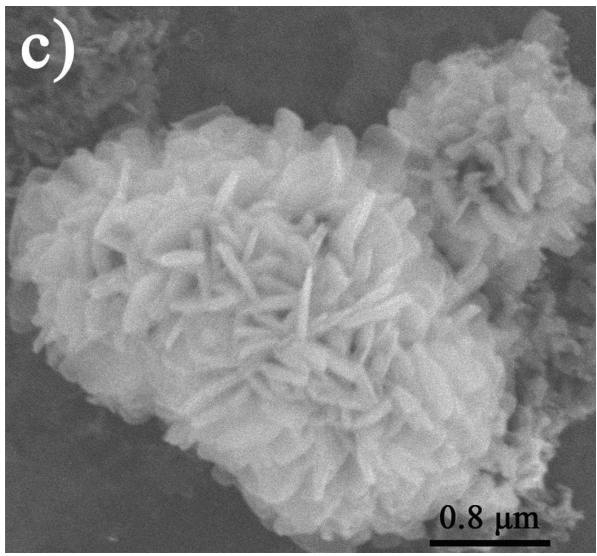

(c)

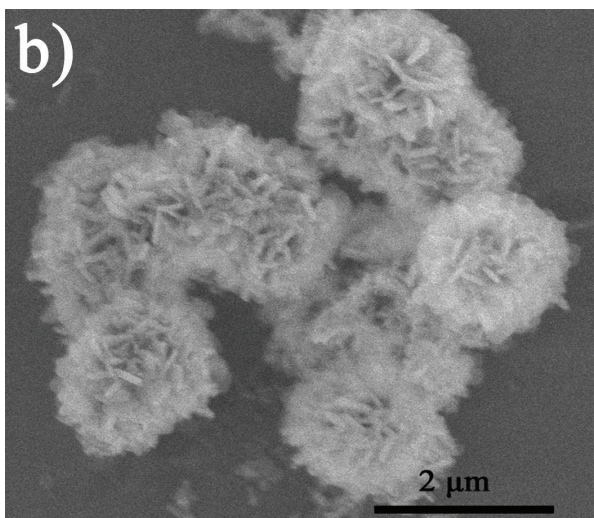

(b)

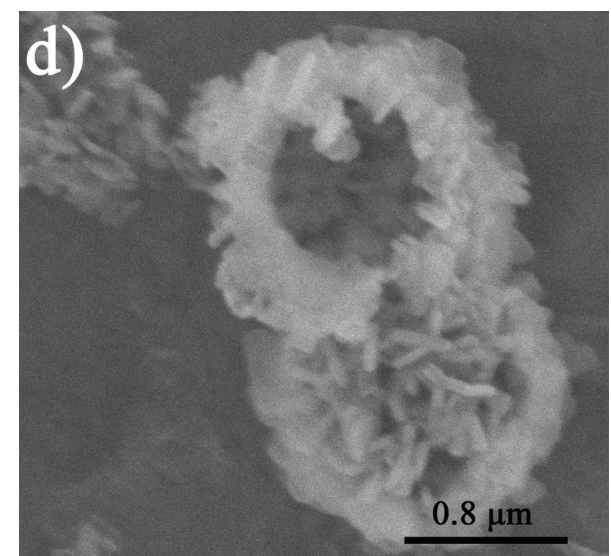

(d)

FIGURE 1: SEM images of sample 1.

also display better luminescent properties than other solid materials.

So far, there have been many researches of organic hollow sphere structures fabrication reported. However, there have been few inorganic nanostructures constructed by the nanosheet [8]. In this paper, we report a general one-pot lowcost solvothermal method for preparation of $\mathrm{Tb}^{3+}$-based $\mathrm{CP}$ nanosheet and construction into aggregates of hollow microspheres (1), using $\mathrm{Tb}^{3+}$ as metal center and 2-methyl benzoic acid (2-MeBAH) as ligand. The formation of nanosheet and spherical hollow structures is unusual since no templates or surfactant is intentionally used during the synthetic process. The downward luminescence of $\mathbf{1}$ was investigated in detail.

\section{Experimental Section}

$\mathrm{Tb}_{4} \mathrm{O}_{7}$ was bought from Ganzhou Kemingrui Rare Earth Company (Ganzhou, China). 2-MeBAH was purchased from Aladdin Company (Shanghai, China) and used as received. $\mathrm{TbCl}_{3} \cdot 6 \mathrm{H}_{2} \mathrm{O}$ was prepared by dissolving $\mathrm{Tb}_{4} \mathrm{O}_{7}(99.9 \%)$ with concentrated $\mathrm{HCl}_{3}$ and then evaporated at $100^{\circ} \mathrm{C}$ until the crystal film formed. Other reagents were from Guangzhou Chemical Reagent Factory (AR, Guangzhou, China) and used without further purification.
In a typical process, $\mathrm{Tb}^{3+}$-based $\mathrm{CP}$ nanosheet and its aggregates of hollow microspheres were prepared as follows: an aqueous solution of 2-MeBAH $(0.1 \mathrm{~g}$ in $25 \mathrm{~mL})$ was added with a $0.1 \mathrm{M} \mathrm{NaOH}$ solution to adjust the ligand solution at $\mathrm{pH}=6$, in a stoichiometric amount to yield the sodium salt in situ. The resulting solution was added dropwise to a $10 \mathrm{~mL}$ $0.1 \mathrm{~g} \mathrm{TbCl}_{3} \mathrm{DMF}$ solution $\left(100^{\circ} \mathrm{C}\right)$. All the ligand solution was added to the salt solution in an hour and resulted in a white precipitate. The solid was separated by centrifugation, then washed with water and ethanol, and dried in vacuum desiccator for several days.

Powder X-ray diffraction (PXRD) was carried out using a Rigaku X-ray diffractometer with $\mathrm{Cu}-\mathrm{K} \alpha$ radiation $(\lambda=$ $1.54178 \AA$ ). Scanning electron microscopy (SEM) was performed on a Hitachi S-3400. Elemental analysis (EA, C and $\mathrm{H}$ ) was performed on an EA3000 elemental analyzer. Downconversion luminescence spectrum was recorded on an FLS 980 (Edinburgh Instruments, UK) at room temperature.

\section{Results and Discussion}

SEM was utilized to characterize the morphology and structure of as-prepared samples. As presented in Figure 1(a), it can be seen that sample 1 consists of well aggregated 


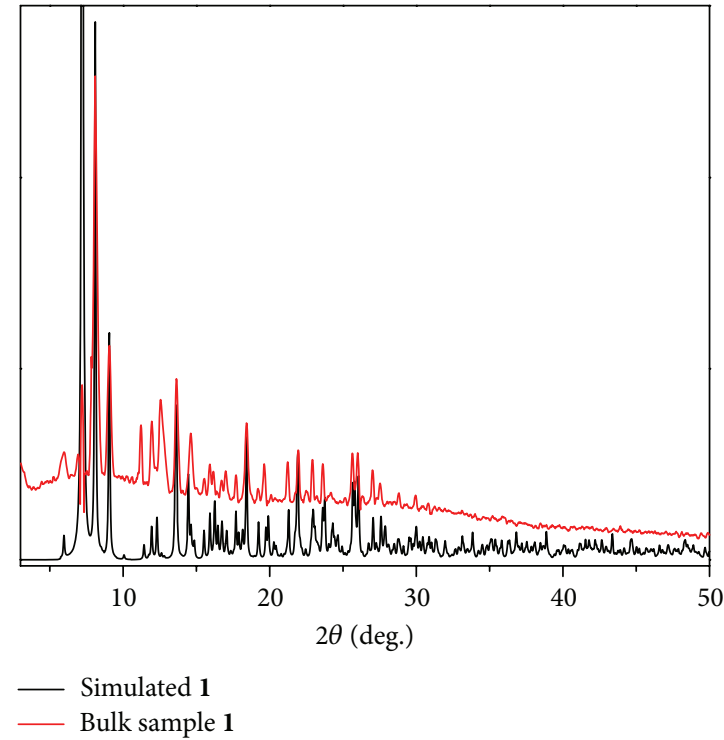

FIgURe 2: PXRD of simulated and bulk sample $\mathbf{1}$.

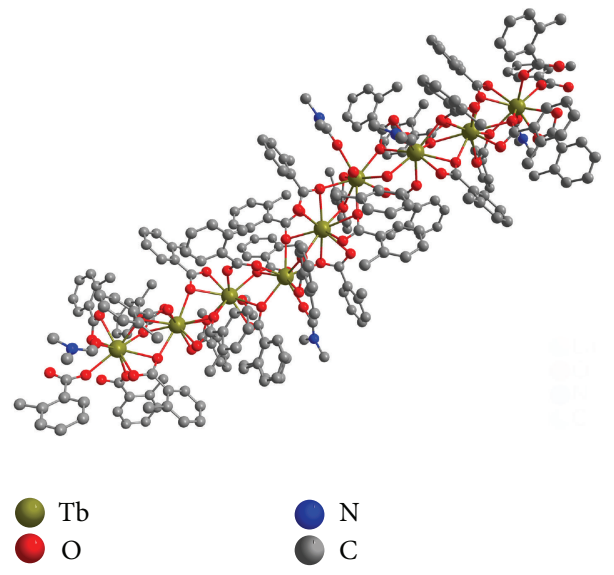

FIgURE 3: The 1D structure of 1 constructed by trinuclear SBUs.

nanosheet, and the aggregated nanosheet forms uniform ball-like structure (Figure 1(b)). Enlargement of the ball-like structure shows that the aggregate units of nanosheets are uniform, with a smooth surface and a narrow size distribution in 50-70 nm (Figure 1(c)).

PXRD of bulk sample 1 in Figure 2 shows evident diffraction peaks, which means that $\mathbf{1}$ is a crystalline material. Further investigation found that the diffraction peaks compete well with the data (CCDC: 634372) reported by Busskamp et al. [14]. The reported result is a lanthanum complex, and in our result, $\mathbf{1}$ may be a terbium complex; this indicates that $\mathbf{1}$ is isostructural to the reported data [14]. Thus, it can be deduced that nanomaterial 1 has the chemical formula of $\mathrm{Tb}_{3}(2$ $\mathrm{MeBAH})_{9}(\mathrm{DMF})_{3}$. It has one-dimensional polymer structure (Figure 3), and the one-dimensional structure is connected by the trinuclear second building unit (SBU, Figure 4); the 1D structure is connected by hydrogen bond and van der Waals (VDW) forth to form 3D structure.

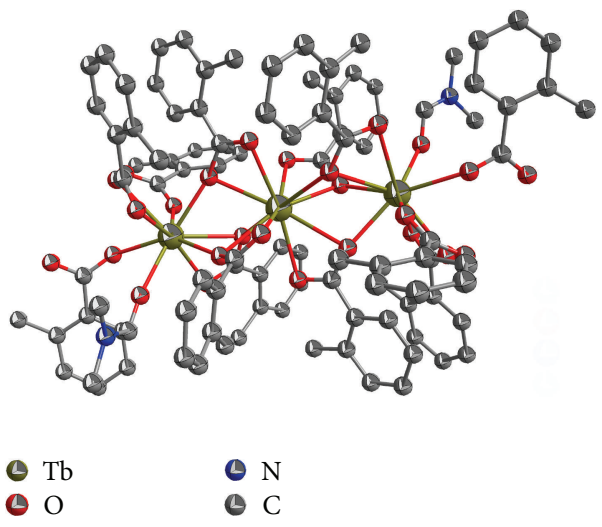

FIgURE 4: The SBU structure of material 1.

The elemental analysis of $\mathbf{1}$ reveals that $\mathrm{C}$ is $51.17 \%$ and $\mathrm{H}$ is $4.151 \%$, which is consistent with the calculated results 


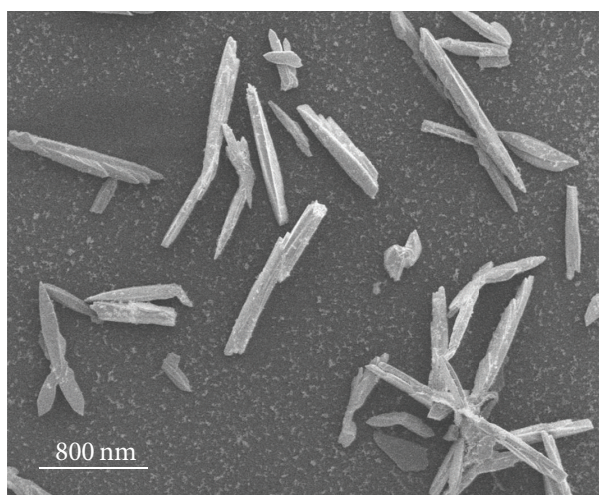

(a)

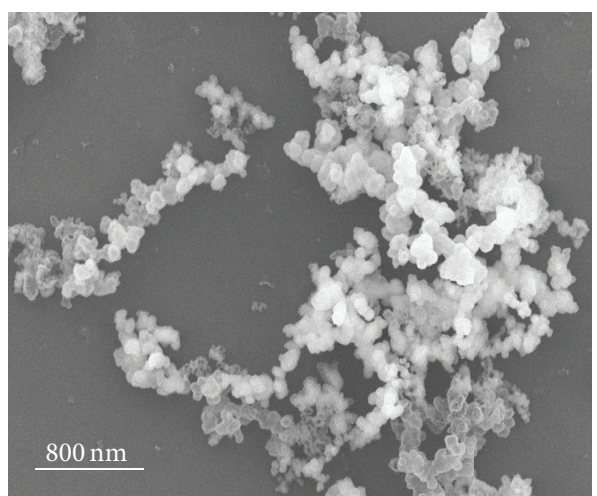

(b)

FIGURE 5: The SEM of different weight ratio of $\mathrm{Tb}^{3+}$ to ligand: (a) $\mathrm{Tb}^{3+} /$ ligand $=1: 3$; (b) $\mathrm{Tb}^{3+} /$ ligand $=3: 1$.

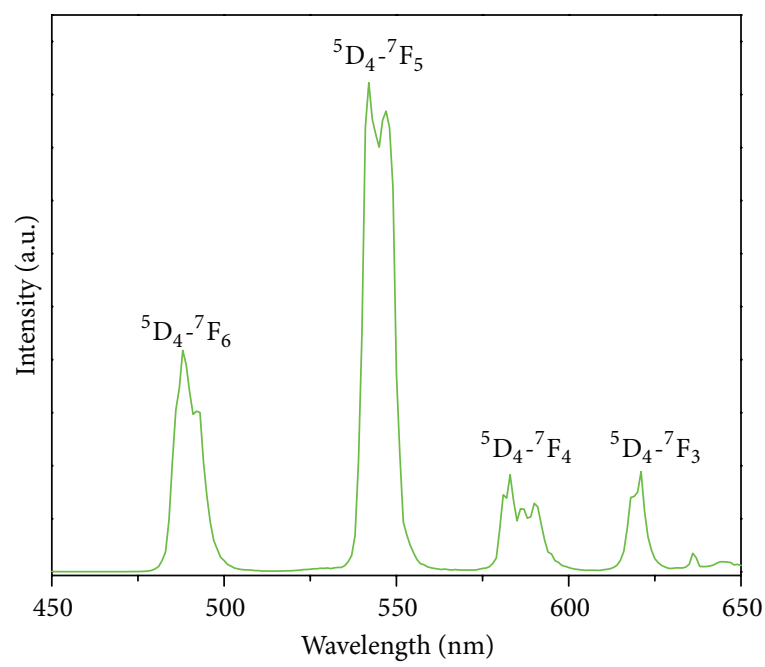

FIgURE 6: Downconversion luminescence spectrum of $\mathbf{1}$.

well (C, 50.94; H, 4.220). This further confirms that bulk nanomaterial really has structure as depicted in Figures 3 and 4 and has the molecular formula of $\mathrm{Tb}_{3}(2-\mathrm{MeBAH})_{9}(\mathrm{DMF})_{3}$.

In order to reveal the factors influencing the formation and morphology of the hollow spheres of $\mathbf{1}$, controlled experiments were carefully performed [15]. As shown by the SEM images in Figure 5, when the weight ratio of $\mathrm{Tb}^{3+}$ to ligand is $1: 3$, it is found that almost all the products are microrods, and when the weight ratio is $3: 1$, the product is ruleless ball-like structure.

Due to the unique properties of rare earth elements, the downward luminescent property of sample 1 was determined at the excitation wavelength of $320 \mathrm{~nm}$ under ambient temperature. Sample 1 exhibits characteristic transitions of the $\mathrm{Tb}^{3+}$ ion at $489,544,583$, and $621 \mathrm{~nm}$, corresponding to the transitions of ${ }^{5} \mathrm{D}_{4} \rightarrow{ }^{7} \mathrm{~F}_{6},{ }^{5} \mathrm{D}_{4} \rightarrow{ }^{7} \mathrm{~F}_{5},{ }^{5} \mathrm{D}_{4} \rightarrow{ }^{7} \mathrm{~F}_{4}$, and ${ }^{5} \mathrm{D}_{4} \rightarrow{ }^{7} \mathrm{~F}_{3}$, respectively (Figure 6) [16-19]. The strongest peak is ascribed to the transition of ${ }^{5} \mathrm{D}_{4} \rightarrow{ }^{7} \mathrm{~F}_{5}[4,20-22]$. The ligand is at the excited ${ }^{1} \pi \pi^{*}$ state after the simultaneous absorption of one photon and then transfers to the excited ${ }^{3} \pi \pi{ }^{*}$ state through intersystem crossing (ISC), after that, the energy transfer (ET) to ${ }^{5} \mathrm{D}_{4}$ state of $\mathrm{Tb}^{3+}$, and luminescence is generated by the ${ }^{5} \mathrm{D}_{4}{ }^{-} \mathrm{F}_{j}(j=6,5,4$ and 3$)$ transitions.

\section{Conclusions}

Lanthanide CPs nanosheet aggregates have been synthesized via a facile template-free solvothermal strategy using DMF as a solvent and 2-MeBAH as ligand. The products were characterized by PXRD, SEM, EA, and downward luminescence. The downward luminescence of product 1 built from $\mathrm{Tb}^{3+}$ and 2-MeBAH has one-dimensional structure connected by trinuclear SBU. Downward luminescence shows that sample 1 exhibits characteristic transitions of ${ }^{5} \mathrm{D}_{4}{ }^{-}{ }^{7} \mathrm{~F}_{j}(j=6,5,4$ and 3) for $\mathrm{Tb}^{3+}$ at $489,544,583$, and $621 \mathrm{~nm}$, respectively, and the strongest peak is at $544 \mathrm{~nm}$ ascribed to the transition of ${ }^{5} \mathrm{D}_{4} \rightarrow{ }^{7} \mathrm{~F}_{5}$.

\section{Competing Interests}

The authors declare that they have no competing interests. 


\section{Acknowledgments}

The authors acknowledge the financial support of the Open Project Program of Key Laboratory of Functional Small Organic Molecule, Ministry of Education (no. KLFS-KF201422) and Jiangxi Normal University (no. 5616).

\section{References}

[1] D. Yang, Y. Wang, Y. Wang, Z. Li, and H. Li, "Luminescence enhancement after adding organic salts to nanohybrid under aqueous condition," ACS Applied Materials and Interfaces, vol. 7, no. 3, pp. 2097-2103, 2015.

[2] N. Ekthammathat, T. Thongtem, A. Phuruangrat, and S. Thongtem, "Synthesis and characterization of $\mathrm{CeVO}_{4}$ by microwave radiation method and its photocatalytic activity," Journal of Nanomaterials, vol. 2013, Article ID 434197, 7 pages, 2013.

[3] D. Falcomer, A. Speghini, G. Ibba et al., "Morphology and luminescence of nanocrystalline $\mathrm{Nb}_{2} \mathrm{O}_{5}$ doped with $\mathrm{Eu}^{3+}$," Journal of Nanomaterials, vol. 2007, Article ID 94975, 5 pages, 2007.

[4] C.-H. Zeng, J.-L. Wang, Y.-Y. Yang et al., "Lanthanide CPs: the guest-tunable drastic changes of luminescent quantum yields, and two photon luminescence," Journal of Materials Chemistry C, vol. 2, no. 12, pp. 2235-2242, 2014.

[5] H. Liu, T. Chu, Z. Rao, S. Wang, Y. Yang, and W.-T. Wong, “The tunable white-light and multicolor emission in an electrodeposited thin film of mixed lanthanide coordination polymers," Advanced Optical Materials, vol. 3, no. 11, pp. 1545-1550, 2015.

[6] M. Xie, Y. Zhu, Y. Yang, and F. Zhao, "Ultrafast dynamics of twophoton absorption related filaments in methanol solution of a Tb(III) complex," Optical Materials, vol. 36, no. 4, pp. 727-731, 2014.

[7] C. B. Han, Y. L. Wang, Y. L. Li, C. M. Liu, and Q. Y. Liu, "Slow magnetization relaxation in a one-dimensional chiral dysprosium-carboxylate compound constructed from the cubic $\mathrm{Dy}_{4}\left(\mu_{3}-\mathrm{OH}\right)_{4}$ clusters," Inorganic Chemistry Communications, vol. 58, pp. 91-94, 2015.

[8] S.-L. Zhong, R. Xu, L.-F. Zhang et al., "Terbium-based infinite coordination polymer hollow microspheres: preparation and white-light emission," Journal of Materials Chemistry, vol. 21, no. 41, pp. 16574-16580, 2011.

[9] X. Kang, D. Yang, P. Ma et al., "Fabrication of hollow and porous structured $\mathrm{GdVO}_{4}: \mathrm{Dy}^{3+}$ nanospheres as anticancer drug carrier and MRI contrast agent," Langmuir, vol. 29, no. 4, pp. 1286-1294, 2013.

[10] R. C. Lv, S. L. Gai, Y. L. Dai, F. He, N. Niu, and P. P. Yang, "Surfactant-free synthesis, luminescent properties, and drug-release properties of $\mathrm{LaF}_{3}$ and $\mathrm{LaCO}_{3} \mathrm{~F}$ hollow microspheres," Inorganic Chemistry, vol. 53, no. 2, pp. 998-1008, 2014.

[11] E. Martinez-Castro, J. Garcia-Sevillano, F. Cusso, and M. Ocana, "Template-free synthesis and luminescent properties of hollow Ln:YOF (Ln = Eu or Er plus Yb) microspheres," Journal of Alloys and Compounds, vol. 619, pp. 44-51, 2015.

[12] Z. Wang, H. Liu, S. Wang, Z. Rao, and Y. Yang, "A luminescent Terbium-Succinate MOF thin film fabricated by electrodeposition for sensing of $\mathrm{Cu}^{2+}$ in aqueous environment," Sensors and Actuators B: Chemical, vol. 220, pp. 779-787, 2015.

[13] T. Chu, H. Liu, Y. Yang et al., "A new dinuclear terbium complex as a novel luminescence probe for detecting amines," Journal of Photochemistry and Photobiology A: Chemistry, vol. 294, pp. 3843, 2014.
[14] H. Busskamp, G. B. Deacon, M. Hilder et al., "Structural variations in rare earth benzoate complexes: part I. Lanthanum," CrystEngComm, vol. 9, no. 5, pp. 394-411, 2007.

[15] J. Huo, L. Wang, E. Irran et al., "Hollow ferrocenyl coordination polymer microspheres with micropores in shells prepared by Ostwald ripening," Angewandte Chemie-International Edition, vol. 49, no. 48, pp. 9237-9241, 2010.

[16] K. Zheng, K.-L. Lou, C.-H. Zeng, S.-S. Li, Z.-W. Nie, and S. Zhong, "Hybrid membrane of agarose and lanthanide coordination polymer: a selective and sensitive $\mathrm{Fe}^{3+}$ sensor," Photochemistry and Photobiology, vol. 91, no. 4, pp. 814-818, 2015.

[17] C.-H. Zeng, X.-T. Meng, S.-S. Xu, L.-J. Han, S. Zhong, and M.-Y. Jia, "A polymorphic lanthanide complex as selective $\mathrm{Co}^{2+}$ sensor and luminescent timer," Sensors and Actuators B: Chemical, vol. 221, pp. 127-135, 2015.

[18] Z.-Q. Yan, X.-T. Meng, R.-R. Su et al., "Basophilic method for lanthanide MOFs with a drug ligand: crystal structure and luminescence," Inorganica Chimica Acta, vol. 432, pp. 41-45, 2015.

[19] S.-S. Li, Z.-N. Ye, S.-S. Xu et al., "Highly luminescent lanthanide CPs based on dinuclear cluster: crystal structure and sensitive Trp sensor," RSC Advances, vol. 5, no. 88, pp. 71961-71967, 2015.

[20] T. T. Shen, Y. Zhang, W. S. Liu, and Y. Tang, "Novel multi-color photoluminescence emission phosphors developed by layered gadolinium hydroxide via in situ intercalation with positively charged rare-earth complexes," Journal of Materials Chemistry C, vol. 3, no. 8, pp. 1807-1816, 2015.

[21] C.-H. Zeng, F.-L. Zhao, Y.-Y. Yang et al., "Unusual method for phenolic hydroxyl bridged lanthanide CPs: syntheses, characterization, one and two photon luminescence," Dalton Transactions, vol. 42, no. 6, pp. 2052-2061, 2013.

[22] C.-H. Zeng, Y.-Y. Yang, Y.-M. Zhu, H.-M. Wang, T.-S. Chu, and S. W. Ng, "A new luminescent terbium 4-methylsalicylate complex as a novel sensor for detecting the purity of methanol," Photochemistry and Photobiology, vol. 88, no. 4, pp. 860-866, 2012. 

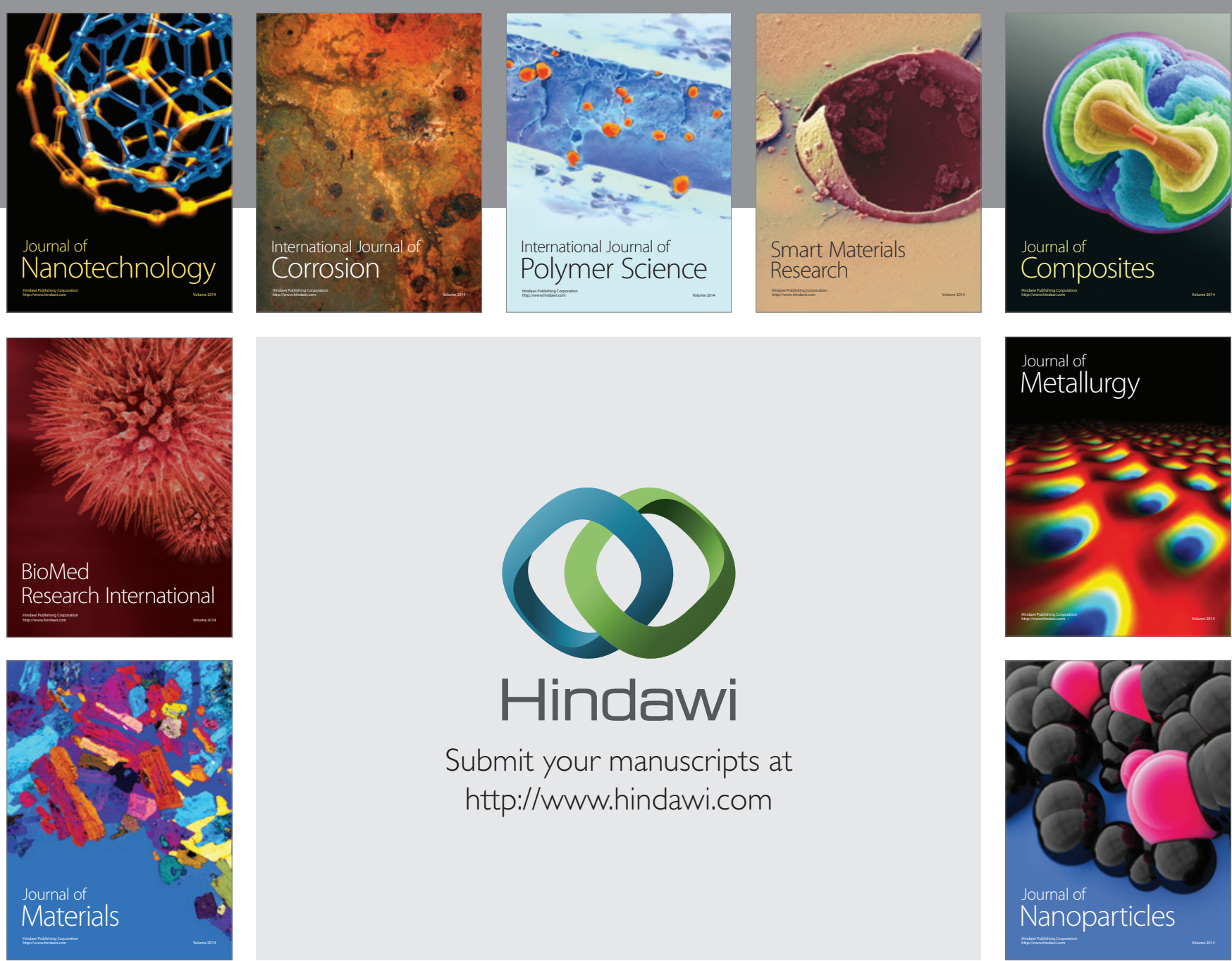

\section{Hindawi}

Submit your manuscripts at

http://www.hindawi.com

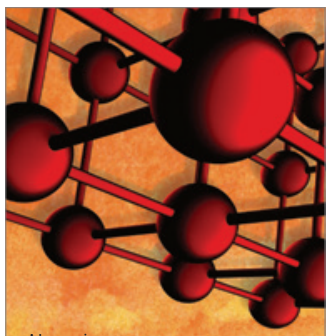

Materials Science and Engineering
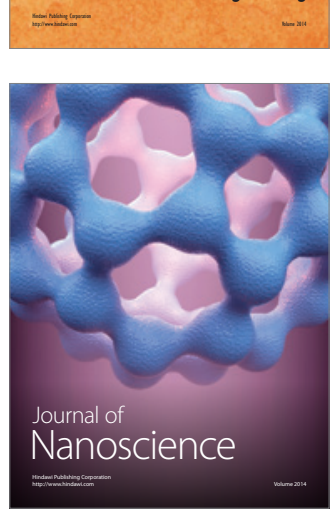
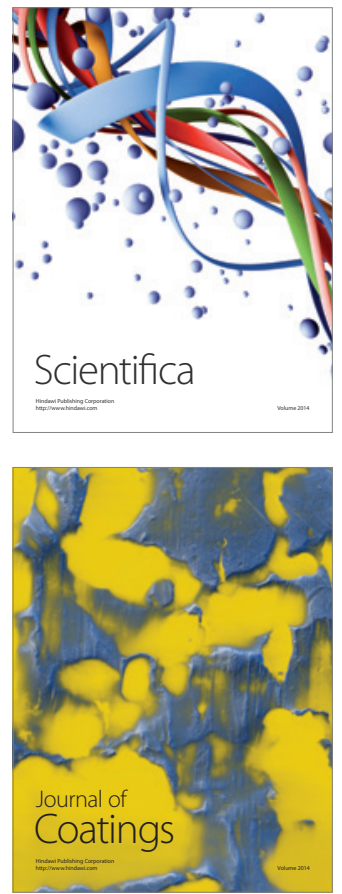
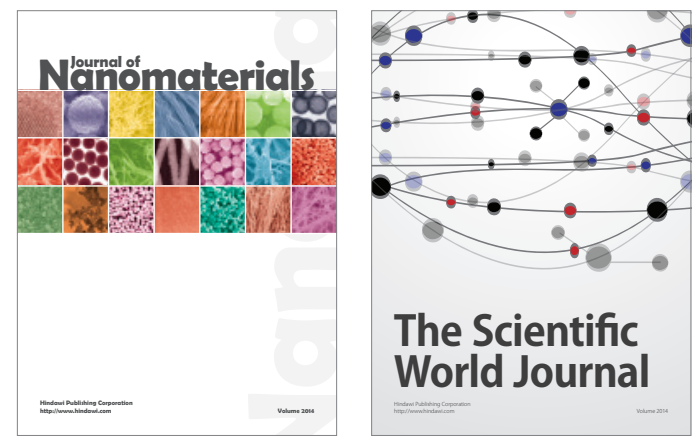

The Scientific World Journal
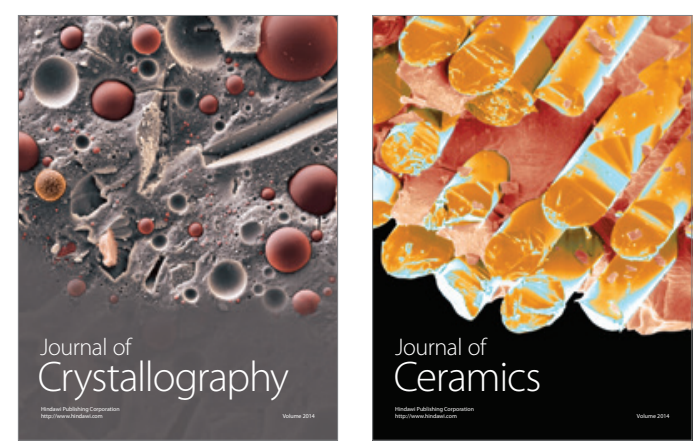
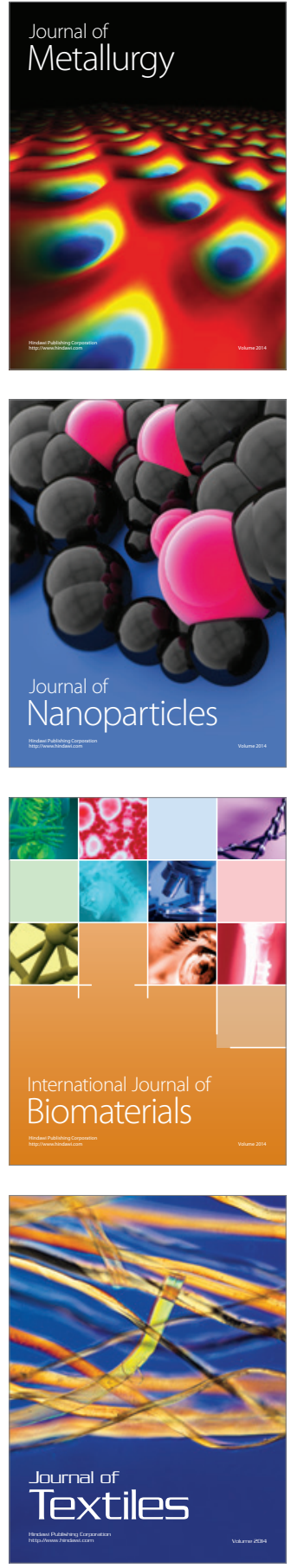